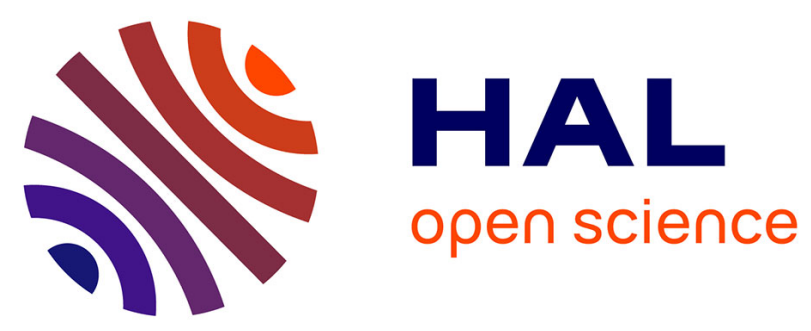

\title{
Space-aware modeling of two-phase electric charging stations
}

\author{
Fabio Antonio Vieira Pinto, Luís Henrique Maciel Kosmalski Costa, Daniel \\ Sadoc Menasche, Marcelo Dias de Amorim
}

\section{- To cite this version:}

Fabio Antonio Vieira Pinto, Luís Henrique Maciel Kosmalski Costa, Daniel Sadoc Menasche, Marcelo Dias de Amorim. Space-aware modeling of two-phase electric charging stations. IEEE Transactions on Intelligent Transportation Systems, 2017, 18 (2), pp.450-459. 10.1109/TITS.2016.2580127 . hal01324106

\section{HAL Id: hal-01324106 https://hal.science/hal-01324106}

Submitted on 31 May 2016

HAL is a multi-disciplinary open access archive for the deposit and dissemination of scientific research documents, whether they are published or not. The documents may come from teaching and research institutions in France or abroad, or from public or private research centers.
L'archive ouverte pluridisciplinaire HAL, est destinée au dépôt et à la diffusion de documents scientifiques de niveau recherche, publiés ou non, émanant des établissements d'enseignement et de recherche français ou étrangers, des laboratoires publics ou privés. 


\title{
Space-aware modeling of two-phase electric charging stations
}

\author{
Fabio Antonio V. Pinto, Luís Henrique M. K. Costa, Daniel S. Menasché, and Marcelo Dias de Amorim
}

\begin{abstract}
In order to match the energy demand of electric vehicles to the capacity of the power grid, it is fundamental to understand the occupancy of charging stations and react accordingly. A Markov model of a fast charging station for Lithium-ion batteries, the most prevalent type today, is proposed. Li-Ion batteries present a two-step charging profile, which makes energy management particularly challenging. A wide range of situations is covered by considering three types of scenarios with and without waiting lines. The analytical results obtained from the steady state solution of the Markov model reveal the behavior of multiple variables of interest: availability of the charging station to accept new customers (in terms of space and energy), number of customers, energy consumption, and power utilization. From the results, indicators for assessing the quality of service $(\mathrm{QoS})$ of the charging station are derived. Based on these indicators, customers may decide to wait or head toward another station. The owners of stations, in turn, can predict the impact of investments in space and energy provisioning, when devising capacity planning strategies.
\end{abstract}

\section{INTRODUCTION}

$\mathbf{S}$ MART CITIES will have to properly plan the geographic distribution of their electric charging stations in a way that drivers do not run out of battery and the power grid does not get overloaded. Determining facility locations for battery loading stations is an NP-hard problem and approximation algorithms require real-world indicators to achieve acceptable results [1]. This imposes a fundamental understanding of the behavior of charging stations.

Modeling charging stations requires taking into account a number of benchmark elements such as available physical space, number of sockets, parking spaces, and their disposition, promoting best uses of the power grid and of charging stations. Existing models consider only a subset of these elements; as a consequence, they provide designers with, at best, a partial view of the system. To circumvent these limitations, a Markov model that captures the key features of fast charging stations is proposed and used to analyze different metrics of interest such as the blocking probability of newcomers as well as the expected waiting time. Such metrics are the most relevant to express the level of Quality of Service (QoS) in charging stations [2]. The particularity of the proposed model is that it considers both the battery charging profile and the physical space of the charging station.

F. A. V. Pinto and L. H. M. K. Costa are with GTA/PEE-COPPE/DELPoli, Federal University of Rio de Janeiro, Brazil. D. S. Menasché is with the Computer Science Department, Federal University of Rio de Janeiro, Brazil. M. D. de Amorim is with LIP6/CNRS, UPMC Sorbonne Universités, Paris, France. Emails: \{fabiovieira, luish\}@gta.ufrj.br, sadoc@dcc.ufrj.br, marcelo.amorim@lip6.fr.

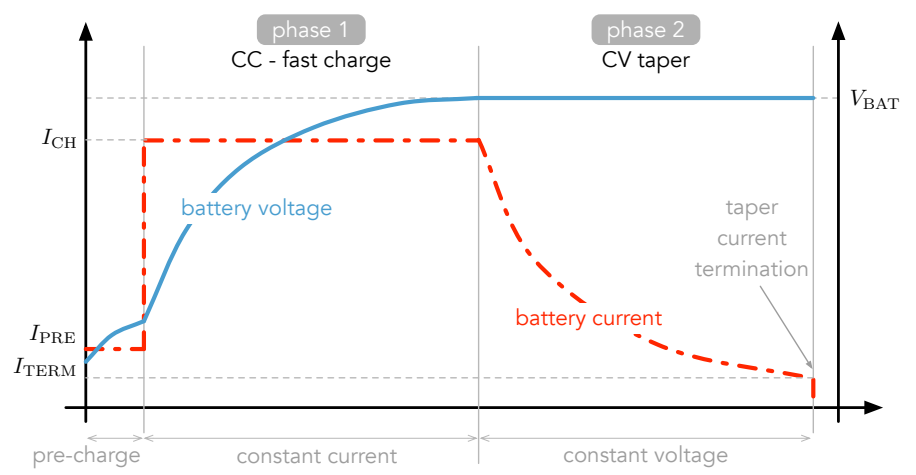

Fig. 1: Lithium-ion charging profile. The pre-charge occurs to prepare the battery to CC-phase with a constant current $\left(I_{\mathrm{CH}}\right)$ until it reaches the nominal voltage $\left(V_{\mathrm{BAT}}\right)$. During the CVphase, the current decays exponentially down to the current termination $\left(I_{\text {TERM }}\right)$ when the process ends.

Broad availability of fast charging stations is required to avoid the well-known problem of range anxiety, i.e., when drivers worry about batteries running out of power before reaching the destination or a charging station. According to [3], $40 \%$ of users exceed the energy capacity storage of EV batteries in their daily trips. Once a station is reached, charging times may vary widely. Charging times of slow charging stations range from 8 to 16 hours. Fast charging stations, in contrast, ensure an average charging time of 30 minutes. This paper considers fast charging stations for lithium-ion (Li-ion) batteries, which are the most prevalent batteries nowadays.

The first challenge in modeling such a system is to consider the charging profile of the battery. The charging profile of Liion batteries is composed of two phases, namely CC (constant current) and CV (constant voltage) [4], [5]. The charging procedure is illustrated in Fig. 1. According to Kester et al. [6] and Linden et al. [7], around 65\% of the total charge is accomplished at CC-phase, whereas the other $35 \%$ occurs during the CV-phase. The proposed model is flexible enough to accommodate these two phases. The second challenge is to take into account the physical space of the charging station. Indeed, whether the station includes or not parking slots has a direct impact on the satisfaction experienced by customers.

This paper proposes a continuous time and discrete state space Markov chain to explain the behavior of a charging 
station, accounting for the charging profile of Li-ion batteries. ${ }^{1}$ The numerical results obtained from the steady state solution of the proposed model reveal properties of several metrics of interest, such as the unavailability of the charging station to accept new customers due to space and energy constraints, the expected number of customers in the station (including the waiting customers) and the power utilization.

As a summary, the contributions of this paper are:

- Analytical model. Our model scrutinizes the charging profile of the Li-ion battery and, with necessary simplifications, suggests a charging station model more detailed than those existing in the literature. Multiple scenarios that vary in terms of parking capacity and expected waiting time are explored.

- Station design. From the viewpoint of the station owners, our analysis assist the design of charging stations, considering the rational use of energy, the size of the station and customer waiting time. Through the knowledge of the average vehicle flow in the region where the station is planned, it is possible to choose the number of sockets to serve the EVs and define the power consumption of the station.

- Support for charging profiles. The charging profile affects the efficient use of available space and energy, impacting customers waiting times and the station power utilization. This work considers the charging profile of Li-ion batteries, but the proposed model can be easily adapted to assess the impact of different charging profiles of future EVs equipped with novel batteries.

This paper is organized as follows. Section II describes related work. Section III formulates the problem and describes the scenarios under consideration. The proposed model is introduced in Section IV, followed by numerical results which are presented in Section V. Section VI discusses the conclusions and possible avenues for future research.

\section{RELATED WORK}

There is a growing literature on the impact of EV charging on the electrical grid, accounting for the smart routing of EVs to available stations. Ban et al. propose a charging station that uses the multi-queue system concept [9]. They investigate how energy consumption reacts when the behavior of EV owners is controlled. The incentive to feed the batteries at lower prices or a penalty to pay more at peak times would influence the behavior of arrivals of vehicles to charging stations.

The use of vehicular communication has also been investigated in the design of electric vehicle charging systems [10]. Vehicles may adopt wireless technology systems that support communication vehicle-to-vehicle (V2V) and vehicle-toinfrastructure (V2I) based on $3 \mathrm{G}$ and $4 \mathrm{G}$ cellular networks and the IEEE 802.11 p protocol. The locations of the charging stations and their availability can be published using vehicular communications, together with the average waiting times towards the stations. In addition, information about the

\footnotetext{
${ }^{1}$ This paper is a significant extension of a companion paper [8]. It now introduces models of much more realistic scenarios that include both a"waiting plugged" mode and parking spaces.
}

current state of the batteries can be used to determine which stations are reachable. The authors assume that each station can provide up to a pre-determined amount of power, which is uniformly split between its sockets.

Bayram et al. account for charging times [11]. They consider an average charging time of 30 minutes in fast charging stations and assume that incoming customers would not be willing to wait. Using a Markov model, they compute the customer blocking probability for different system parameters. Wang et al. extend this idea by foreseeing a widespread adoption of fast charging stations [12]. They consider a charging station in a public parking campus, where the behavior of customers is highly erratic. In order to relieve the stress to the power grid due to such a non conventional load and provide satisfactory quality of service levels, they propose an auxiliary battery storage to supply customers demand at peak hours. The authors tackle this problem a stochastic control problem based on a continuous-time Markov chain to determine the amount of auxiliary battery storage to be used.

Wang et al. propose a charging plan for EVs to prevent overloading the power grid [13]. They consider a smart grid architecture with real-time communication capabilities. This architecture focuses on mobility aspects by exploring resources of vehicular ad hoc networks for real-time communications between roadside units (RSUs) and electric vehicles. The network disseminates two kinds of information, namely energy conditions of the charging stations and charging state of batteries. The charging scheduling problem is formulated as a time-coupled mixed-integer linear programming (MILP).

\section{TARGET SCENARIOS, CHARGING PROFILES, AND ASSUMPTIONS}

In this paper, a charging station has $s$ sockets with total energy capacity to support up to $m$ concomitant vehicles charging at CC-phase. The exact capacity of the station logically depends on the contract its owner has with the electricity utility, which is an input variable in the proposed model. Moreover, charging stations rely only on the energy they receive from the grid: energy storage systems, such as batteries or ultracapacitors, are outside the scope of this paper. Each socket is associated to a parking spot, i.e., in front of each socket there is space for a single car. As an approximation, and without loss of generality, this work assumes that the CVphase consumes half the energy of the CC-phase. The next assumption is that a vehicle always starts at the CC-phase, switches to the CV-phase, then leaves the station. Together, the two latter assumptions imply that the station has at most $2 m-1$ vehicles at any point in time at CV-phase. Therefore, except otherwise noted, in the reference setup the station has $s=2 m-1$ sockets.

\section{A. Charging profile}

Two aspects of the charging procedure form a baseline of our analysis. The first one is related to the time and power requirements to fill the batteries. This work considers fast chargers at public charging stations. Those consume significant power from the grid but take less time to charge the vehicles 


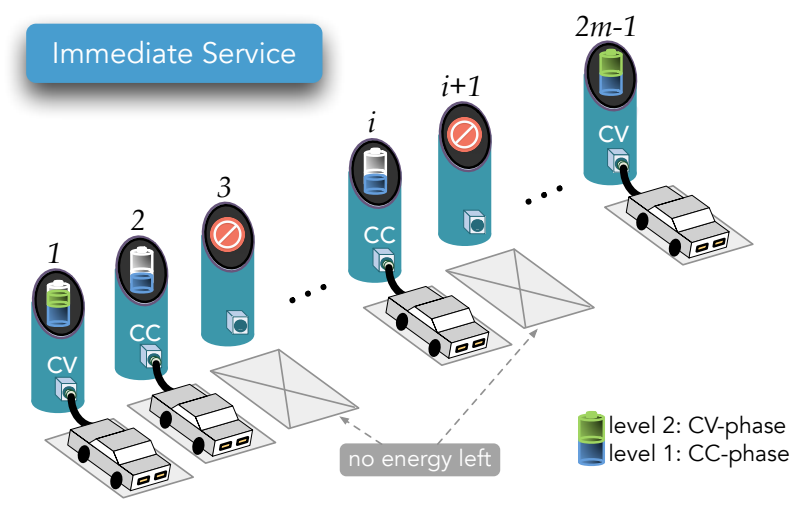

Fig. 2: Immediate Service Scenario. An EV is only admitted into the charging station if there is enough energy to immediately serve it. In the illustrated scenario, the charging station has $2 m-1$ sockets and energy capacity to serve up to $m$ concomitant vehicles at CC-phase.

compared, for instance, to residential charging counterparts. Fast charging mode favors mobility allowing the vehicles to continue their journeys after brief stops at charging stations. The second aspect is related to the particular charging profile of Li-ion batteries as depicted in Fig. 1. In the present analyses, the pre-charge phase is neglected, because it is only triggered when the battery is totally depleted, which only occurs in case of infrequent utilization.

The charging procedure starts at CC-phase. When the nominal voltage at the battery terminals is reached, the CV-phase starts and the current decays exponentially (see again Fig. 1). The CV-phase eliminates voltage drops in resistive elements. In this paper a $24 \mathrm{kWh}$ battery is considered, as used for example by Nissan Leaf, a $100 \%$ electric vehicle. This is also the average battery size of most electric vehicles in market today. The fast charging procedure lasts about half an hour. For security reasons, when the battery reaches $80 \%$ of its capacity, the fast charging process stops and the stored energy is around $19 \mathrm{kWh}$. Approximately $51.2 \mathrm{~kW}$ of power is consumed during the CC-phase (in a period of 15 minutes) and $25.6 \mathrm{~kW}$ during the remaining 15 minutes ( $\mathrm{CV}$-phase).

\section{B. Target scenarios}

Three charging station scenarios are considered here. In the first one, the vehicle will only be admitted into the system if it can be immediately served. In the other two scenarios, it is assumed that the driver may wait before the station is able to supply energy to the EV. These scenarios are detailed next.

Immediate Service Scenario. Fig. 2 illustrates the structure of the charging station in the Immediate Service Scenario. In the Immediate Service Scenario, a vehicle enters the station if it can immediately plug into a socket and start charging. Vehicles are admitted when the station has free sockets and available energy to supply to new EVs. Otherwise, vehicles are blocked. In this scenario there is no queuing.

Plugged Wait Scenario. In this scenario, vehicles may wait in case there is not enough energy by the time they arrive

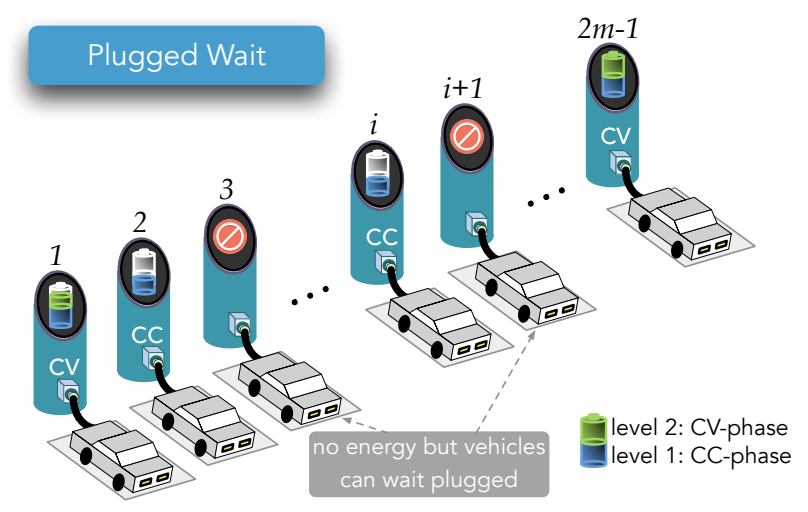

Fig. 3: Plugged Wait Scenario. In this scenario, an EV is admitted into the charging station if it is willing to wait until there is enough energy to start its service. Some cars waiting can be seen in charging spots with a "prohibited parking" sign.

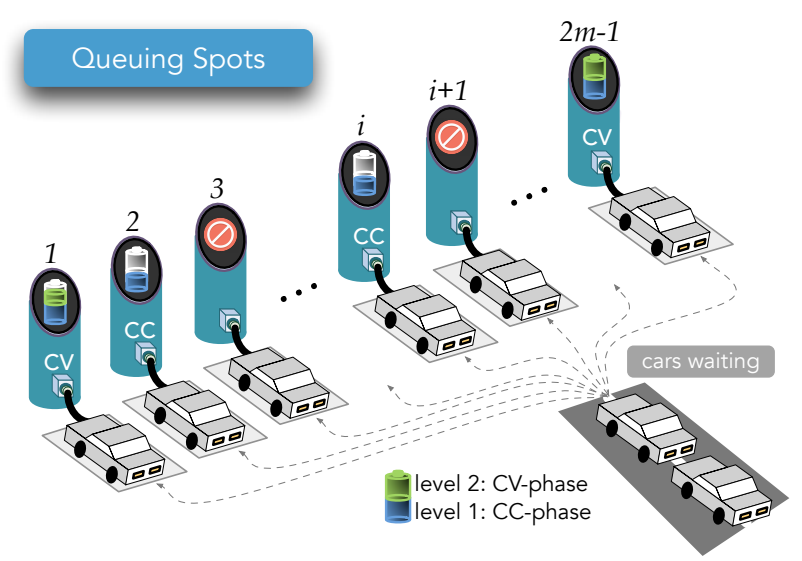

Fig. 4: Queuing Spots Scenario. Besides being able to wait plugged (as in the Plugged Wait Scenario), vehicles also have additional parking spaces at their disposal.

at a station. In the Plugged Wait Scenario, illustrated in Fig. 3, when a vehicle arrives at the charging station and some charging socket is empty, the vehicle is admitted even if the available energy is not sufficient to immediately serve the newcomer. In this case, the vehicle enters a "plugged wait mode" until it can be served. In other words, idle sockets can be used as waiting spots. From the station owner point of view, this system is more profitable than the first one because less customers are declined.

Queuing Spots Scenario. In the Queuing Spots Scenario (Fig. 4), a parking lot is considered where vehicles can wait before plugging into a charging socket. The charging station has more physical space than in the previous two scenarios; such extra space is made available through a number of "queuing spots". As soon as a socket becomes free, the first vehicle that arrived at the parking space can plug and wait to be charged. This scenario admits more vehicles than the two previous scenarios but is associated to longer delays. 


\section{MARKOV MODEL}

The charging station is modeled as a continuous-time Markov chain with discrete state space. Vehicles arrive to the station according to a Poisson process with rate $\lambda$ vehicles/hour. The model assumes that vehicles arrive at the charging station with the battery almost depleted, but not empty. Under this assumption, vehicles which are admitted into the station go through the $\mathrm{CC}$ and $\mathrm{CV}$ phases, but not through the pre-charge phase (Fig. 1). The time to fill the battery in a fast charging station is around 30 minutes [14]. The analytic model assumes that the service times at $\mathrm{CC}$ and $\mathrm{CV}$ phases are exponentially distributed with rates $\mu_{1}$ and $\mu_{2}$, respectively. Except otherwise noted, it also assumes that $1 / \mu_{1}=1 / \mu_{2}=15$ minutes. Table I summarizes the notation used throughout this paper.

Recall that the three scenarios described in Section III are associated to different queuing strategies. While the Immediate Service Scenario admits no waiting before service, the Plugged Wait Scenario may have vehicles waiting in idle sockets and in the Queuing Spots Scenario there might be vehicles in a parking courtyard. Let $K$ be the number of parking spaces in the parking courtyard. In the Immediate Service Scenario and in the Plugged Wait Scenario, $K=0$.

The energy that a charging station is capable to supply normalized by the energy consumed by each car at the CVphase is referred to as the Normalized Total Energy Capacity (NTEC), and denoted by $C$. In our reference setup, $C$ equals the capacity of the station divided by $25.6 \mathrm{~kW}$. For example, a station where $C=8$ may have up to 4 vehicles charging at the CC-phase or 7 vehicles charging at the CV-phase. Note that although the station has energy capacity to support up to 8 vehicles at the $\mathrm{CV}$-phase, such state is not reachable, as vehicles always start charging at the $\mathrm{CC}$-phase, and only leave the system after going through the two phases.

\section{A. State Space Description}

This section presents the Markov model to characterize the behavior of the charging station. Let $N_{c c}, N_{c v}$ and $N_{w}$ be random variables characterizing the number of vehicles in the CC-phase, CV-phase and waiting line, respectively. The realizations of the random variables are denoted by the respective lower case letters. Let $\boldsymbol{\sigma}=\left(n_{c c}, n_{c v}, n_{w}\right)$ be the system state. The admitted vehicles are divided between plugged and unplugged. Let $n_{p}$ and $n_{u}$ be the number of plugged and unplugged vehicles, respectively. Thus, $n_{c c}+n_{c v}+n_{w}=$ $n_{p}+n_{u}$. While all vehicles in CC and CV phases are plugged to sockets, some of the waiting vehicles might be plugged whereas others wait in parking spots (if the scenario allows). Let $P$ be the normalized power consumed by the system, $P=2 n_{c c}+n_{c v}$.

First, consider the Immediate Service and the Plugged Wait Scenarios, depicted in Fig. 5 (remembering that $C=2 m$ and $s=2 m-1$ ). The Immediate Service Scenario is fully represented by plane 0 , comprising light-green and light red states, for which $n_{w}=0$. In plane 0 , the light-green states have available energy and space to admit new vehicles. The
TABLE I: Table of Notation

\begin{tabular}{c|l|l}
\hline Variable & Description & Observation \\
\hline$N_{c c}$ & Number of cars at CC-phase. & \\
$N_{c v}$ & Number of cars at CV-phase. & \\
$N_{w}$ & Number of cars waiting. & \\
$N$ & Number of cars. & $N=N_{p}+N_{u}$ \\
$N_{p}$ & Number of cars plugged. & \\
$N_{u}$ & Number of cars unplugged. & $N_{p}+N_{u}=N_{c c}+N_{c v}+N_{w}$ \\
$P$ & Normalized power consumed. & $P=2 N_{c c}+N_{c v}$ \\
\hline Parameter & Description & Default value \\
\hline$s$ & Number of sockets. & $2 m-1$ \\
$K$ & Number of parking spaces. & $m-1$ \\
$C$ & Normalized power capacity & $2 m$ \\
\hline Metric & (also referred to as NTEC). & \\
\hline$p_{b}$ & Description & Observation \\
$p_{c 1}$ & Plocking probability. & \\
& Probability of being admitted & \\
$p_{c 2}$ & Probability of being admitted & \\
& and waiting. & $p_{c 1}+p_{c 2}=1-p_{b}$ \\
$T_{w}$ & Waiting time. & $E\left[T_{w}\right]=E\left[N_{w}\right] /\left(\lambda\left(1-p_{b}\right)\right)$ \\
\hline
\end{tabular}

light-red states in plane 0 , in contrast, have no available energy to admit new vehicles - these states are energy constrained.

In the Plugged Wait Scenario, vehicles can wait plugged into sockets $\left(n_{w} \geq 0\right.$ and $\left.n_{u}=0\right)$. Thus, the Plugged Wait Scenario is represented by plane 0 together with higher order planes in Fig. 5. Let $h$ be the index of the highest plane, i.e., $h$ is the maximum number of vehicles waiting to be served at any point in time. As the number of sockets is $s=2 m-1$, and that the station has capacity to serve $m$ vehicles at CC-phase, the maximum number of vehicles that could wait plugged is $h=m-1$. Vehicles that arrive at the energy constrained scenarios plug in a socket and wait. That is represented in Fig. 5 by blue, violet, orange, and green states, for $n_{w}$ equal to $1, h-2, h-1$ and $h$, respectively.

Consider the states at the right edge of Fig. 5, that is, states $(m-i, 2 i, h-i)$ and $(m-i-1,1+2 i, h-i)$, for $i=0, \ldots, h$. These states are blocking states which means that no vehicles can be admitted into the system.

Table II fully characterizes the state transitions for the Queuing Spots Scenario, which generalizes the other two scenarios. When the conditions presented in the first column are satisfied, arrivals and services occur at the rates specified in the second column, leading to a transition to the state described in the third column. After the transition, variables $n_{p}, n_{u}$ and $P$ are updated according to the rules specified in the fourth, fifth and sixth columns, respectively.

Let $\pi$ be the system steady state solution vector, $\pi=$ $\left(\pi_{0,0,0}, \pi_{0,0,1}, \ldots, \pi_{m, 0, h+K}\right)$. Let $\Omega$ be the system state space. When distinguishing between the Immediate Service, Plugged Wait and Queuing Spot Scenarios, the state spaces are noted as $\Omega_{I S}, \Omega_{P W}$ and $\Omega_{Q S}$, respectively.

Let $\mathbf{Q}$ be the infinitesimal generator matrix. Then, $\pi \mathbf{Q}=\mathbf{0}$ and $\sum_{(i, j, k) \in \Omega} \pi_{i, j, k}=1$.

\section{B. Metrics of Interest}

The metrics used to measure the behavior of the charging station in the three scenarios are: 


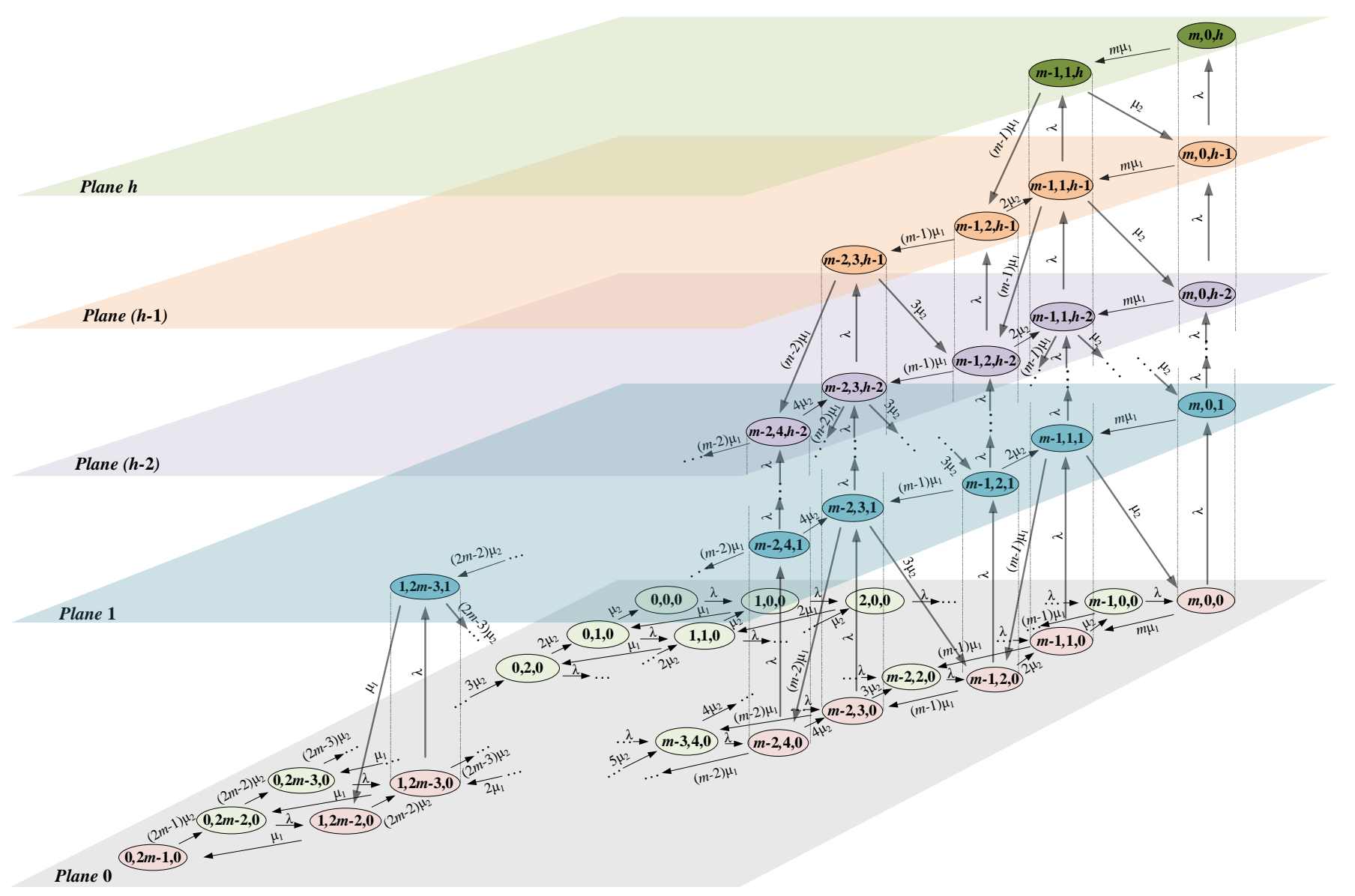

Fig. 5: Markov chain of a fast charging station. States in the chain are represented by the tuple $\left(n_{c c}, n_{c v}, n_{w}\right)$.

TABLE II: State transitions of the Markov chain from state $\left(n_{c c}, n_{c v}, n_{w}\right)$.

\begin{tabular}{|c|c|c|c|c|c|}
\hline \multirow{2}{*}{ Condition } & \multirow{2}{*}{ Rate } & \multirow{2}{*}{ Next State } & \multicolumn{3}{|c|}{ Additional State Update } \\
\hline & & & $n_{p}$ & $n_{u}$ & $P$ \\
\hline $\begin{array}{l}\text { Service at CC-phase not followed by start of CC service: } \\
\left(n_{c c}>0\right) \& \&\left(\left(n_{w}=0\right) \| !((P+1) \leq C)\right)\end{array}$ & $n_{c c} \mu_{1}$ & $\left(n_{c c}-1, n_{c v}+1, n_{w}\right)$ & $n_{p}$ & $n_{u}$ & $P-1$ \\
\hline $\begin{array}{l}\text { Service at CC-phase followed by start of CC service: } \\
\left(n_{c c}>0\right) \& \&\left(n_{w}>0\right) \& \&(P+1) \leq C\end{array}$ & $n_{c c} \mu_{1}$ & $\left(n_{c c}, n_{c v}+1, n_{w}-1\right)$ & $n_{p}$ & $n_{u}$ & $P+1$ \\
\hline $\begin{array}{l}\text { Service at CV-phase followed by neither start of CC service nor new vehicle socket plugin: } \\
\left(n_{c v}>0\right) \& \& !\left(n_{w}>0 \& \& P+1 \leq C\right) \& \& !\left(n_{u}>0\right)\end{array}$ & $n_{c v} \mu_{2}$ & $\left(n_{c c}, n_{c v}-1, n_{w}\right)$ & $n_{p}-1$ & $n_{u}$ & $P-1$ \\
\hline $\begin{array}{l}\text { Service at CV-phase followed by new vehicle socket plugin but no new CC service: } \\
\left(n_{c v}>0\right) \& \& !\left(n_{w}>0 \& \& P+1 \leq C\right) \& \&\left(n_{u}>0\right)\end{array}$ & $n_{c v} \mu_{2}$ & $\left(n_{c c}, n_{c v}-1, n_{w}\right)$ & $n_{p}$ & $n_{u}-1$ & $P-1$ \\
\hline $\begin{array}{l}\text { Service at CV-phase followed by start of CC service but no new vehicle socket plugin: } \\
\left(n_{c v}>0\right) \& \&\left(n_{w}>0 \& \& P+1 \leq C\right) \& \& !\left(n_{u}>0\right)\end{array}$ & $n_{c v} \mu_{2}$ & $\left(n_{c c}+1, n_{c v}-1, n_{w}-1\right)$ & $n_{p}-1$ & $n_{u}$ & $P+1$ \\
\hline $\begin{array}{l}\text { Service at CV-phase followed by start of CC service and new vehicle socket plugin: } \\
\left(n_{c v}>0\right) \& \&\left(n_{w}>0 \& \& P+1 \leq C\right) \& \&\left(n_{u}>0\right)\end{array}$ & $n_{c v} \mu_{2}$ & $\left(n_{c c}+1, n_{c v}-1, n_{w}-1\right)$ & $n_{p}$ & $n_{u}-1$ & $P+1$ \\
\hline $\begin{array}{l}\text { Arrival and immediate service: } \\
\left(n_{p}<s\right) \& \&(P+2) \leq C\end{array}$ & $\lambda$ & $\left(n_{c c}+1, n_{c v}, n_{w}\right)$ & $n_{p}+1$ & $n_{u}$ & $P+2$ \\
\hline $\begin{array}{l}\text { Arrival being plugged without immediate service: } \\
\left(n_{p}<s\right) \& \&(P+2)>C\end{array}$ & $\lambda$ & $\left(n_{c c}, n_{c v}, n_{w}+1\right)$ & $n_{p}+1$ & $n_{u}$ & $P$ \\
\hline $\begin{array}{l}\text { Arrival without being plugged: } \\
\left(s \leq n_{p}\right) \& \&\left(n_{p}+n_{u}<s+K\right)\end{array}$ & $\lambda$ & $\left(n_{c c}, n_{c v}, n_{w}+1\right)$ & $n_{p}$ & $n_{u}+1$ & $P$ \\
\hline
\end{tabular}

Blocking probability $\left(p_{b}\right)$. The probability of a vehicle being rejected because the system has reached its maximum capacity. It is split in three blocking probabilities:

- Due to lack of space $\left(p_{b 1}\right)$. There is no empty socket or parking space for the arriving vehicle.

- Due to lack of energy $\left(p_{b 2}\right)$. There is no available energy to start charging the arriving vehicle.

- Due to lack of space and of energy $\left(p_{b 3}\right)$. There is neither space nor energy available for the arriving vehicle.

Note that $p_{b}=p_{b 1}+p_{b 2}+p_{b 3}$. Let $\mathcal{S}_{b}$ be the set of states for which the system is blocked. Let $\mathcal{S}_{b 1}, \mathcal{S}_{b 2}$ and $\mathcal{S}_{b 3}$ be the sets of states for which the system is blocked due to space, due to energy, and due to space and energy, respectively. We use superscripts $I S, P W$, and $Q S$ to refer to the sets in the Immediate Service, Plugged Wait, and Queuing Spot Scenarios, respectively. Then, $p_{b \ell}=\sum_{(i, j, k) \in \mathcal{S}_{b \ell}} \pi_{i, j, k}$, for $\ell=1,2,3$.

The numerical evaluations consider the special case, where $s=2 m-1$. For such setting, in the Immediate Service 
Scenario, $\mathcal{S}_{b 1}^{(I S)}=\emptyset$, and

$$
\begin{aligned}
\mathcal{S}_{b 2}^{(I S)} & =\left\{\boldsymbol{\sigma} \in \Omega_{I S}: n_{p}^{\boldsymbol{\sigma}}<s \text { and }\left(P^{\boldsymbol{\sigma}}+2\right)>C\right\} . \\
\mathcal{S}_{b 3}^{(I S)} & =\left\{\boldsymbol{\sigma} \in \Omega_{I S}: n_{p}^{\boldsymbol{\sigma}}=s \text { and }\left(P^{\boldsymbol{\sigma}}+2\right)>C\right\} \\
& =\{(0,2 m-1,0),(1,2 m-2,0)\} .
\end{aligned}
$$

In the Plugged Wait Scenario, for $s=2 m-1$ the subset $\mathcal{S}_{b 1}^{(P W)}=\mathcal{S}_{b 2}^{(P W)}=\emptyset, p_{b}^{(P W)}=p_{b 3}^{(P W)}$, and

$$
\mathcal{S}_{b 3}^{(P W)}=\left\{\boldsymbol{\sigma} \in \Omega_{P W}: n_{p}^{\boldsymbol{\sigma}}=s \text { and }\left(P^{\boldsymbol{\sigma}}+2\right)>C\right\} .
$$

In the Queueing Spot Scenario, for $s=2 m-1$ and $K=m-1$, the subset $\mathcal{S}_{b 1}^{(Q S)}=\mathcal{S}_{b 2}^{(Q S)}=\emptyset, p_{b}^{(Q S)}=p_{b 3}^{(Q S)}$, and

$$
\begin{aligned}
\mathcal{S}_{b 3}^{(Q S)}=\left\{\boldsymbol{\sigma} \in \Omega_{Q S}:\left(n_{p}^{\boldsymbol{\sigma}}+n_{u}^{\boldsymbol{\sigma}}\right)=(s+K)\right. \text { and } \\
\\
\left.\left(P^{\boldsymbol{\sigma}}+2\right)>C\right\} .
\end{aligned}
$$

Note also that $\mathcal{S}_{b 3}^{(I S)} \subset \mathcal{S}_{b 3}^{(P W)}$ and $\left|\mathcal{S}_{b 3}^{(P W)}\right|=\left|\mathcal{S}_{b 3}^{(Q S)}\right|$.

Probability of being immediately served $\left(p_{c 1}\right)$. The probability that the charging station has space and available energy to immediately charge the arriving vehicle. In the Immediate Service Scenario, $p_{c 1}=1-p_{b}$, i.e., if the system is not blocked, it receives the vehicle and immediately charges its battery. Thus, $1-p_{b}$ is the probability of accepting vehicles in the system. In the other two scenarios, the EV can be admitted in idle sockets and parking spaces and can thus wait before being served. In the Immediate Service scenario, once a vehicle is accepted, it starts charging immediately; thus, in the other scenarios, $p_{c 1} \leq 1-p_{b}$.

Probability of being admitted and waiting $\left(p_{c 2}\right)$. The probability that the charging station has available space to admit the arriving vehicle, but no energy to immediately charge it. Note that $p_{c 1}+p_{c 2}=1-p_{b}$. This equation means that the probability of being admitted or served is equal to the probability of not being rejected by the system.

Expected number of vehicles in the system $(E[N])$. The expected number of vehicles in the station is a reward obtained from the Markov model. A reward of a given state is the value of the state times the stationary probability of the state. Thus, the total reward of a charging station is the sum of the product over $(i, j, k, \ell)$ of the probability of each state by the number of vehicles at CC-phase $(i)$ plus the number of vehicles at CVphase $(j)$ plus the number of vehicles waiting plugged and in the parking spaces $(k, \ell)$. For Immediate Service scenario, $k=0$ and $\ell=0$; for the Plugged Wait scenario, $\ell=0$. Thus, the general equation is given by

$$
E[N]=\sum_{(i, j, k, \ell) \in \Omega} \pi_{i, j, k, \ell}(i+j+k+\ell) .
$$

Let $E\left[N_{w}\right]$ be the expected number of EVs that wait for available energy, plugged to a socket or in parking spaces. Similarly to the previous equation, the number of vehicles waiting is given by

$$
E\left[N_{w}\right]=\sum_{(i, j, k, \ell) \in \Omega} \pi_{i, j, k, \ell} \cdot(k+\ell) .
$$

Expected normalized power consumption $(E[P])$. The expected normalized power consumption is the sum of twice the number of vehicles at CC-phase plus the number of vehicles at CV-phase (reflecting the double energy demand of the CCphase) times the probability of each state. Thus, it is given by

$$
E[P]=\sum_{(i, j, k, \ell) \in \Omega}(2 i+j) \cdot \pi_{i, j, k, \ell}
$$

Power utilization $\left(E\left[P_{\%}\right]\right)$. The ratio between the expected normalized power consumption and the normalized power capacity of the station, $E\left[P_{\%}\right]=E[P] / C$.

Expected waiting time $\left(E\left[T_{w}\right]\right)$. The expected waiting time before service is obtained using Little's law, $E\left[T_{w}\right]=$ $E\left[N_{w}\right] /\left(\lambda\left(1-p_{b}\right)\right)$.

\section{Evaluation Results}

In this section, the numerical results are reported. The goal is to perform sensitivity analysis of different system metrics, such as expected waiting time and power utilization, with respect to the three model parameters: a) vehicle arrival rate, b) station energy capacity, and c) number of sockets. This analysis can assist the owner of the charging station to decide whether to invest in energy or space improvements. In our numerical evaluations, the Tangram-II tool is used to automatically generate and solve the proposed Markov chain, and to obtain the metrics of interest [15]. The results of some important variables, such as blocking probability, number of vehicles, and power utilization were generated by Tangram. To confirm the validity of the exponential variables assumed for the $\mathrm{CC}$ and $\mathrm{CV}$ service times in the analytical model, simulation in Tangram-II [15] was used with deterministic service times for both CC and CV. The analytical and simulation results obtained with Tangram are plotted in the same graph. The simulation results are an average over 12 simulation runs, with $10^{6}$ events each. Confidence intervals of $95 \%$ are represented by vertical errorbars in the graph (often in the graphs small errorbar collapses to an horizontal trace). The bars with the light-blue edge contour represent analytical results. As one can see, the behavior of the simulation results corroborate the analytical results.

\section{A. Reference Setup}

Table III summarizes the input parameters used in the evaluation, which are motivated by [16], [17]. The vehicle arrival rate $\lambda$ varies from 5 to 50 vehicles/hour. The number of sockets $s$ varies from 3 to 17 , in steps of 2 . Finally, except otherwise noted, the capacity $C=s+1$.

\section{B. Numerical Results}

1) Blocking probability $\left(p_{b}\right)$ : Fig. 6 shows how the blocking probability varies as a function of the vehicle arrival rate, for different values of $s$. The blocking probability sharply increases for $\lambda$ varying between 5 and 20 vehicles/hour. For $\lambda>20$ vehicles/hour, the increase is smoother. Note also that 
TABLE III: Parameters used in the evaluation of charging stations.

\begin{tabular}{c|l|c} 
Parameter & Description & Values \\
\hline$\lambda$ & Vehicle Arrival Rate (vehicles/hour) & 5 to 50 \\
$\mu$ & Total Service Rate (vehicles/hour) & 2 \\
$\mu_{1}$ & Service Rate at CC-phase (vehicles/hour) & 4 \\
$\mu_{2}$ & Service Rate at CV-phase (vehicles/hour) & 4 \\
$s$ & Number of sockets & 3 to 17 \\
$K$ & Number of parking spaces & 0 to 8 \\
$C$ & Normalized power capacity & 4 to 18
\end{tabular}

for $\lambda=5$ vehicles/hour, the differences between the Immediate Service, Plugged Wait and Queuing Spots Scenarios are most significant. For $\lambda=50$ vehicles/hour, in contrast, the three scenarios behave very similarly. For instance, if $\lambda=50$ and $s=3, p_{b}$ equals $0.9120,0.9089$ and 0.9077 in the three scenarios, respectively.
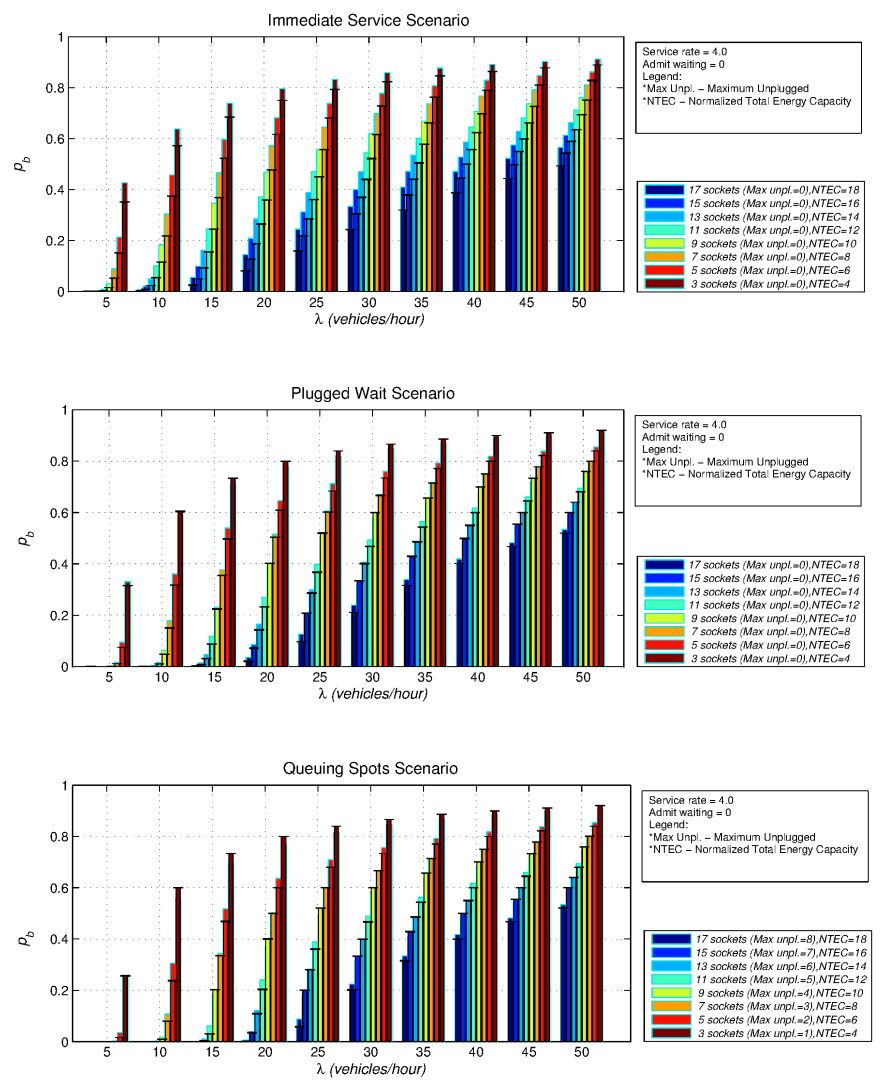

Fig. 6: Blocking probability $\left(p_{b}\right)$.

1.a) Blocking probability due to lack of space $\left(p_{b 1}\right)$ : Given that the station has $s=2 m-1$ sockets and $C=2 m$ capacity, the probability that a vehicle arrives at the station and finds an empty socket but no energy available is $p_{b 1}=0$. Note that the model can also be used to evaluate scenarios where $s<C-1$, and in those cases $p_{b 1} \geq 0$.

1.b) Blocking probability due to lack of energy $\left(p_{b 2}\right)$ : The probability, $p_{b 2}$, is plotted as a function of $\lambda$ for the Immediate Service Scenario in Fig. 7 (in Plugged Wait and Queuing Spots Scenarios, $p_{b 2}=0$ for the parameters of the reference setup). As the arrival rate increases, $p_{b 2}$ increases.

Note that $p_{b 2}$ increases with respect to $s$ and decreases with respect to $C$. Recall that the reference setup assumes that the energy grows linearly with respect to the number of sockets, i.e., $C=s+1$. For arrival rates smaller than 20 vehicles/hour, as $s$ and $C$ are concomitantly increased, the impact of $C$ dominates and $p_{b 2}$ decreases. However, for $\lambda \geq 20$ vehicles/hour note that $p_{b 2}$ first increases and then decreases. The joint increase of the number of sockets, $s$, from 3 to 5 sockets and of the normalized total energy capacity (NTEC), $C$, from 4 to 6 units causes an increase in $p_{b 2}$ and a decrease in $p_{b 3}$. The net effect is a reduction in $p_{b}=p_{b 2}+p_{b 3}$ (see Fig. 6).

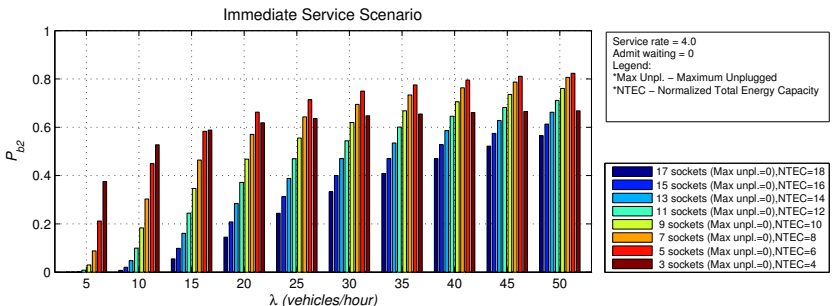

Fig. 7: Blocking probability due to lack of energy in Immediate Service Scenario $\left(p_{b 2}\right)$.

1.c) Blocking probability due to lack of space and energy $\left(p_{b 3}\right)$ : Fig. 8 shows how $p_{b 3}$ varies as a function of the number

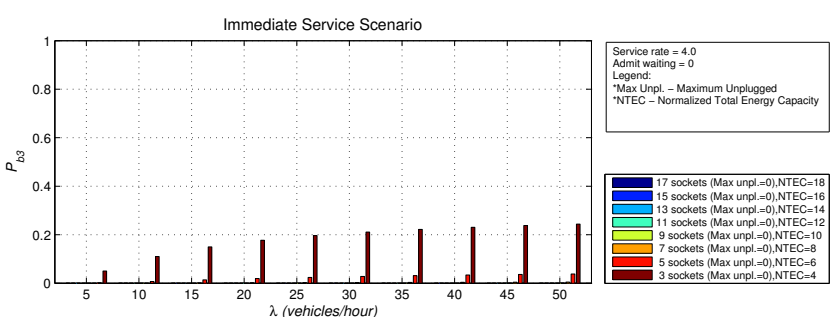

Fig. 8: Blocking probability due to lack of space and energy $\left(p_{b 3}\right)$.

of sockets, arrival rate and available energy. Note that in the Immediate Service Scenario $p_{b 3}$ is small compared to the other two scenarios. This is because in this scenario there are only two states for which vehicles are blocked due to space and energy, i.e., $\left|\mathcal{S}_{b 3}^{(I S)}\right|=2$ and $p_{b 3}^{(I S)}=\pi_{(0,2 m-1,0)}+\pi_{(1,2 m-2,0)}$ (see Eq. (1)). It is interesting to remember that, in Plugged Wait and Queuing Spots scenarios, $\mathcal{S}_{b 1}=\mathcal{S}_{b 2}=\emptyset$, thus, $p_{b}=$ $p_{b 3}$. Thus, the Fig. 8 only shows the $p_{b 3}$ for Immediate Service scenario. In the Plugged Wait and Queuing Spots Scenarios, $p_{b 3}$ (shown as $p_{b}$ in Fig. 6) is roughly the same when $\lambda \geq 20$. This occurs because parking spots rapidly fill up for large values of $\lambda$, and $\left|\mathcal{S}_{b 3}^{(P W)}\right|=\left|\mathcal{S}_{b 3}^{(Q S)}\right|$ (see Section IV-B).

2) Probability of being immediately served $\left(p_{c 1}\right)$ : Fig. 9 shows how $p_{c 1}$ varies as a function of different system parameters. Note that $p_{c 1}$ assumes larger values in the Immediate Service Scenario, at the expense of more vehicles being rejected compared against the other two scenarios. In the Plugged Wait Scenario, for $\lambda \geq 25$ vehicles/hour, as the number of sockets $s$ increases and the relationship $C=s+1$ is maintained, $p_{c 1}$ first decreases and then increases. This is because the initial increase in $s$ reduces $p_{b}$, but increases the chance that vehicles have to wait before being charged (i.e., 

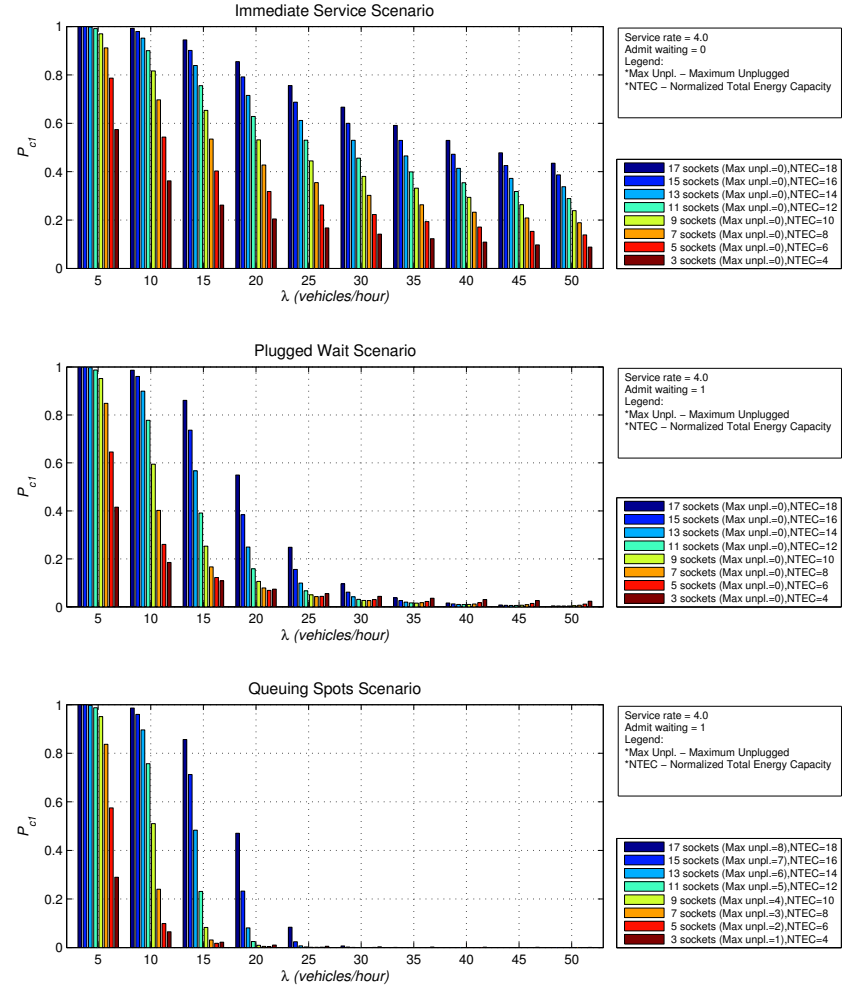

Fig. 9: Probability of being immediately served $\left(p_{c 1}\right)$.
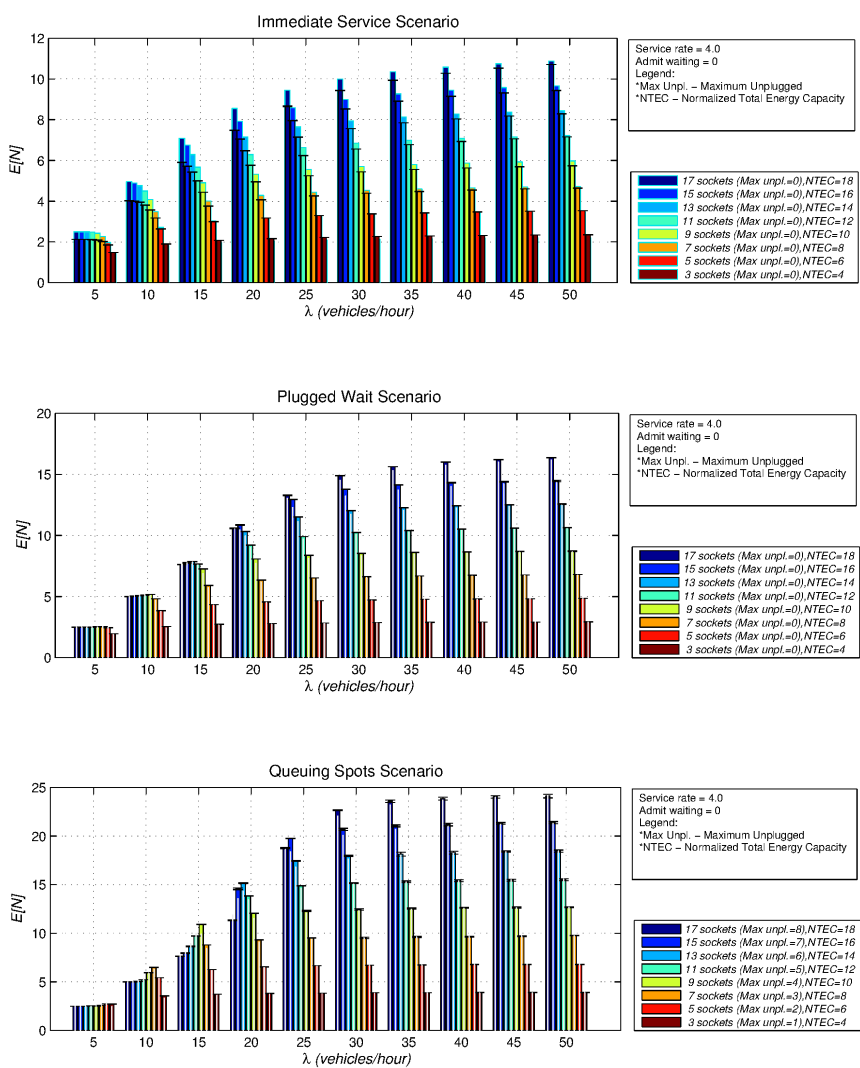

Fig. 10: Number of vehicles in the charging station $(E[N])$.

$p_{c 2}$ increases). Recall that $p_{c 1}+p_{c 2}=1-p_{b}$. This indicates that even though the increase of $s$ always causes a beneficial decrease in $p_{b}$, it might also lead to a detrimental decrease in $p_{c 1}$ at the cost of an increase in $p_{c 2}$.
3) Expected number of vehicles $(E[N])$ : Fig. 10 shows how the expected number of vehicles varies a function of different system parameters. The longer the vehicles are allowed to wait, the larger the expected number of vehicles in the system. In particular, the Queuing Spots Scenario is associated to the largest expected occupancy.

Next, the Plugged Wait Scenario is considered, with $s=17$ sockets. For $\lambda \geq 35$ vehicles/hour the graph shows that $E[N] \geq 15$. When $\lambda=50$ vehicles/hour, $E[N]=16.17$. In this case, the 17 sockets are almost always full. To overcome the high utilization of the station, switching to the Queuing Spots Scenario with additional 8 queuing spots $(K=8)$ is considered. As Fig. 10 shows, for $\lambda=50$ vehicles/hour, $s=17$ sockets and $K=8$ additional queuing spots, the expected number of vehicles in the station under the Queuing Spots Scenario is 24.17 vehicles, again very close to the station capacity. This indicates that to reduce the blocking probability of the station under high arrival rates, the investments in energy and space provisioning must be carefully studied.

Consider now the Queuing Spots Scenario with $\lambda$ varying between 5 and 20 vehicles/hour. As the number of sockets and the energy capacity increase the expected number of vehicles in the station first increases and then decreases. This is due to the balance between space and energy availability. Note that the expected number of clients in the system, $E[N]$, increases with respect to the number of sockets $s$ (space) and decreases with respect to the station power capacity $C$ (energy). Therefore, concomitantly increasing $s$ and $C$ yields a growth followed by a decay in $E[N]$, for $\lambda$ varying between 5 and 20 vehicles/hour (Fig. 10).

Fig. 11 shows the expected number of vehicles in the station as a function of the number of sockets $(s)$ and of the normalized total energy capacity (NTEC), $C$. For any given value of s (respectively, $C$ ), $E[N]$ decreases (respectively, increases) monotonically with respect to $C$ (respectively, $s$ ). The white dotted line indicates the points for which the relation $C=s+1$ holds, and can be used to analyze the scenario in which $s$ and $C$ increase concomitantly, as in Fig. 10. Next, consider only the points over the white dotted line, each point corresponds to a bar in Fig. 10. The initial increase in $s$ from 2 to 3 yields a significant increase in the occupancy of the station, which means that at this region the space constraint plays a significant role. However, as space and energy are further increased, the expected number of vehicles eventually starts to decrease. This is because the increase in energy capacity favors a reduction in the expected number of vehicles in the system, and in the expected number of vehicles waiting in line, as shown in Table IV.

Table IV shows the expected number of vehicles in the waiting line, for the Plugged Wait and Queuing Spots Scenarios, respectively. Consider, for instance, the setup where $\lambda=20$ vehicles/hour. As the number of sockets increases the expected number of waiting vehicles first increases and then decreases. As pointed out in the previous paragraphs, this occurs because the energy capacity of the station increases linearly with respect to the number of sockets. Note that if the charging time is reduced due to future technological advances, the number of sockets that maximizes the expected number 


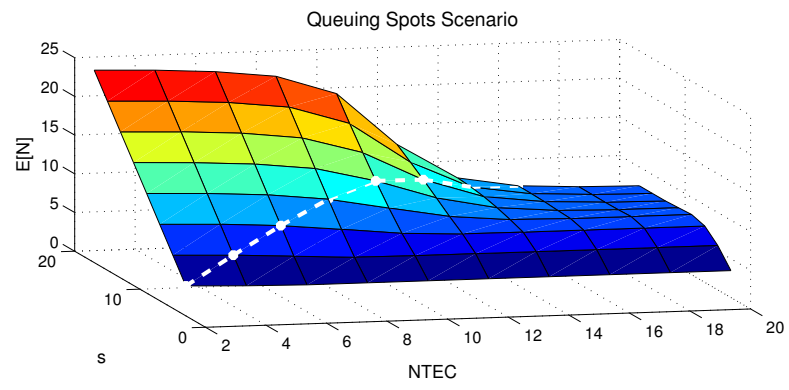

Fig. 11: Expected number of vehicles $E[N]$ in the Queuing Spots Scenario for $\lambda=15$ vehicles/hour, varying the number of sockets and NTEC.

TABLE IV: Expected number of waiting vehicles $\left(E\left[N_{w}\right]\right)$.

\begin{tabular}{c|c|c|c|c|c}
\hline Scenarios & $\lambda$ & $s=5$ & $s=9$ & $s=13$ & $s=17$ \\
\hline \multirow{3}{*}{ Plugged Wait } & 10 & 0.6 & 0.4 & 0.1 & 0.0 \\
\cline { 2 - 6 } & 15 & 0.8 & 1.1 & 0.7 & 0.2 \\
\cline { 2 - 6 } & 20 & 1.0 & 1.6 & 1.6 & 0.9 \\
\hline \multirow{3}{*}{ Queuing Spots } & 10 & 1.9 & 1.0 & 0.1 & 0.0 \\
\cline { 2 - 6 } & 15 & 2.6 & 3.8 & 1.5 & 0.2 \\
\cline { 2 - 6 } & 20 & 2.8 & 5.2 & 5.6 & 2.0 \\
\hline
\end{tabular}

of waiting vehicles will change. Table IV indicates that the proposed model can be used by a station planner to decide how to invest the available budget in energy and space expansions, accounting for the impact of its decisions on the expected number of customers waiting in line and, consequently, on the expected waiting times.

4) Power utilization $\left(E\left[P_{\%}\right]\right)$ : Fig. 12 shows how the power utilization varies as a function of different system parameters. For $\lambda \geq 45$ vehicles/hour, the maximum power utilization for the three scenarios occurs when the number of sockets equals 17. For lower arrivals rates, the power utilization first increases and then decreases as a function of the number of sockets and the energy capacity of the station. The initial increase is due to the larger number of expected vehicles in the station. The final decrease is due to the fact that after a certain point, the utilization of the station is more sensible to the increase in energy capacity. Note also that for smaller arrival rates the three scenarios are more distinguished. When the arrival rate increases, the differences in the fraction of power used between the three scenarios are very subtle.

5) Expected waiting time in line $\left(E\left[T_{w}\right]\right)$ : The expected waiting time in line is an important metric from the point of view of the customers. After being admitted into the station, it can be used by the clients to decide if they must stay inside the car or if they have time for a coffee, or to make a cash withdrawal. Fig. 13 shows that for small stations (few number of sockets) and low arrival rates, clients should not leave their vehicles.

Consider the Plugged Wait Scenario, with $\lambda=50$ vehicles/hour and $s=17$. Then, $E\left[T_{w}\right]=11.65$, i.e. clients might consider stretching their legs before being served. In contrast, when $\lambda=5$ vehicles/hour and $s=5$ sockets, the waiting time is 3.92 minutes, i.e. drivers must stay inside the car under penalty of losing their turn. Similar behavior occurs in the Queuing Spots Scenario. For $\lambda=50$ vehicles/hour and $s=17$, $E\left[T_{w}\right]=32.20$ minutes. For $\lambda=5$ vehicles/hour and $s=5$,
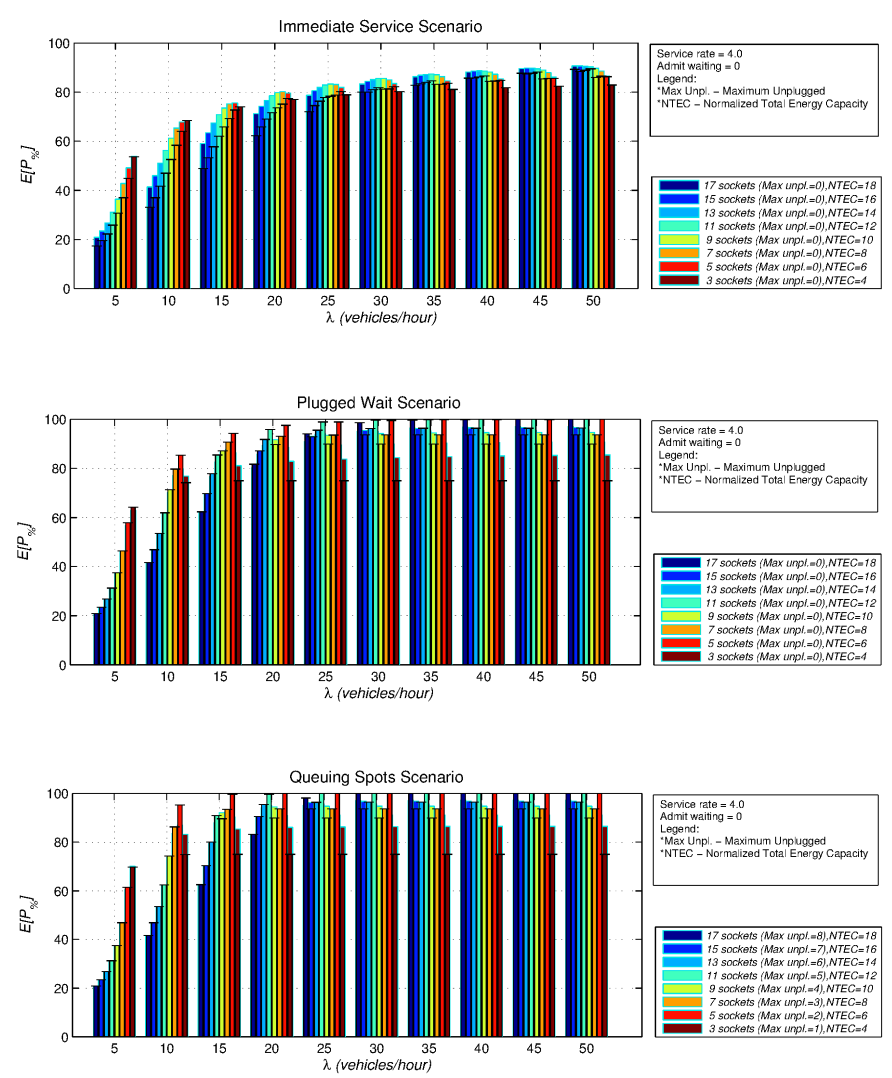

Fig. 12: Percentage power used in the charging stations $\left(E\left[P_{\%}\right]\right)$.
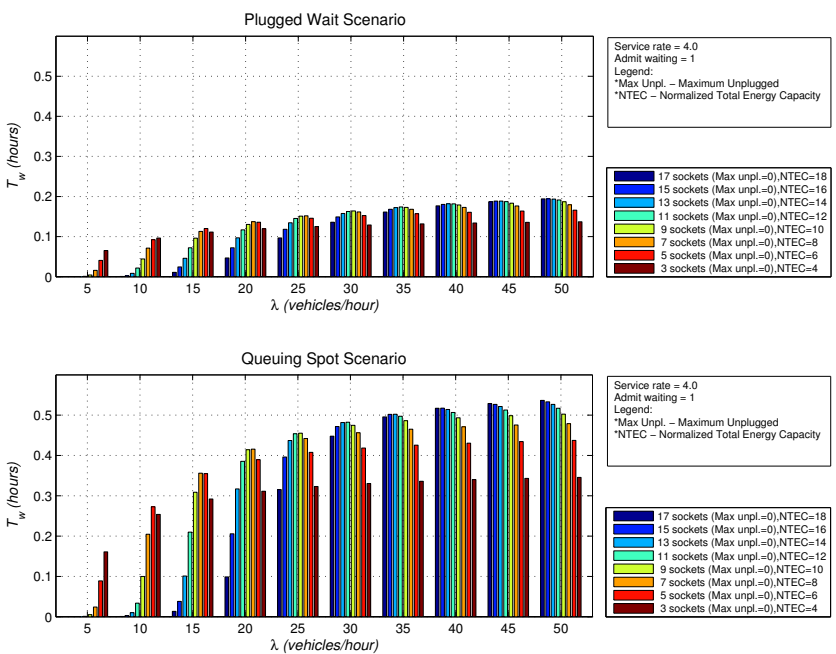

Fig. 13: Expected Waiting time in the System $\left(\left[T_{w}\right]\right)$.

$E\left[T_{w}\right]=9.66$ minutes - tight time to take a coffee.

6) Expected number of vehicles with utilization: Fig. 14 plots the expected number of vehicles as a function of the utilization of the station $(\rho)$. The plot considers the Queuing Spots scenario and three charging station sizes: 3, 9 and 15 sockets, with 1, 4, and 7 parking spaces, respectively. As the arrival rate increases, the utilization of the station also increases and the number of vehicles tends to the space capacity of the station. For a Queuing Spots station with 3 sockets, when $\rho=0.77, E[N]=3.9$. For a station with 


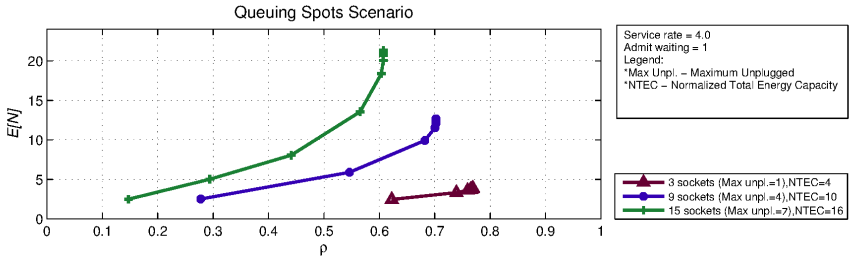

Fig. 14: Number of vehicles versus utilization $(\rho)$.

9 sockets, when $\rho=0.70, E[N]=12.71$. Finally, for a station with 15 sockets, when $\rho=0.61, E[N]=21.33$. The results show that the simulated range of arrival rates covers the maximum capacity of the station, as the number of vehicles almost reach the physical maximum of 4,13 , and 22 vehicles, for all three station sizes, respectively. The utilization of the system $(\rho)$ never reaches 1 because some sockets cannot be used due to energy capacity constraints.

\section{CONCLUSION}

This paper pointed out the peculiar charging profile of lithium-ion batteries as a paramount element to assist in the capacity planning of charging stations. To this goal, a Markov chain model was proposed which, given the workload model and the space and energy capacity of a station, provides important metrics, such as its availability, number of users, power utilization and customers expected waiting time. Such metrics are helpful to the owners of the stations, to the drivers, and to the utilities. Different scenarios in terms of the space available to park electrical vehicles and the expected time that drivers are willing to wait before charging was considered. In the first scenario, no spaces other than the charging spots are available. The EV starts charging as soon as it arrives at the station or it is rejected. In the other two scenarios, drivers may wait. The number of drivers that wait depends on the number of queuing spots in the station, which in turn is a function of the available physical space. Finally, with the results, one can conclude that the design of a fast station must account for the available physical space, the target expected waiting times, and the aimed QoS perceived by the customers. In all scenarios, a smartphone application may assist drivers to find the closest stations which are more likely to admit a vehicle. Devising algorithms to issue routing recommendations making use of the proposed model to predict the state of a station given its current conditions is subject for future work.

The wide adoption of electric vehicles depends on governmental actions to encourage the installation of fast charging stations. The widespread deployment of stations indicate to citizens that electric vehicles are a viable option for short daily trips. The Norwegian Electric Vehicle Association (NEVA), for instance, is pushing the investment on fast charging stations with at least 6 sockets each [16], [17]. The models proposed in this paper are intended as a first step towards a better understanding of the dynamics of vehicles in fast charging stations. Future work consists of parameterizing the fast charging station models proposed in this work using real data, such as from the Netherlands, in order to infer the number and the size of the charging stations needed to serve the fleet.

\section{REFERENCES}

[1] S. Storandt and S. Funke, "Enabling e-mobility: Facility location for battery loading stations," in AAAI Conference on Artificial Intelligence, Bellevue, WA, USA, Jul. 2013.

[2] S. Bayram, G. Michailidis, M. Devetsikiotis, S. Bhattacharya, A. Chakrabortty, and F. Granelli, "Local energy storage sizing in plugin hybrid electric vehicle charging stations under blocking probability constraints," in Architectures and Models for the Smart Grid (IEEE SmartGridComm), Atlanta, GA, Dec. 2011, pp. 78 - 83.

[3] S. Bayram, G. Michailidis, I. Papapanagiotou, M. Devetsikiotis, and F. Granelli, "Decentralized control of electric vehicles in a network of fast charging stations," in Global Communications Conference (GLOBECOM), 2013 IEEE, Atlanta, GA, Dec. 2013, pp. 2785 - 2790.

[4] E. Inoa and J. Wang, "PHEV charging strategies for maximized energy saving," IEEE Transactions on Vehicular Technology, vol. 60, no. 7, pp. 2978-2986, 2011.

[5] Y. Hyok-min, K. Jong-hyun, and S. Eui-ho, "Design of a novel $50 \mathrm{kw}$ fast charger for electric vehicles," Journal of Central South University, vol. 36, no. 4, pp. 372-377, 2013.

[6] W. Kester and J. Buxton, "Practical design techniques for power and thermal management - Section 5: Battery chargers." [Online]. Available: http://www.analog.com/media/en/training-seminars/ design-handbooks/ptmsect5.pdf

[7] D. Linden and T. B. Reddy, Handbook of Batteries. McGraw-Hill, 1995, ch. Chapter 35 - Lithium-Ion Batteries.

[8] F. A. V. Pinto, L. H. M. K. Costa, and M. D. de Amorim, "Modeling spare capacity reuse in EV charging stations based on the Li-ion battery profile," in International Conference on Connected Vehicles \& Expo (ICCVE), Nov. 2014, pp. 92-98.

[9] D. Ban, G. Michailidis, and M. Devetsikiotis, "Demand response control for PHEV charging stations by dynamic price adjustments," in IEEE PES Innovative Smart Grid Technologies (ISGT), Jan. 2012, pp. 1-8.

[10] A. Hess, F. Malandrino, M. B. Reinhardt, C. Casetti, K. A. Hummel, and J. M. Barcelo-Ordinas, "Optimal deployment of charging stations for electric vehicular networks," in First workshop on Urban networking (UrbaNe), Dec. 2012, pp. 1-6.

[11] S. Bayram, G. Michailidis, M. Devetsikiotis, and F. Granelli, "Electric power allocation in a network of fast charging stations," IEEE Journal on Selected Areas in Communications, vol. 31, no. 7, pp. 1235-1246, Mar. 2013.

[12] Q. Wang, I. S. Bayram, F. Granelli, and M. Devetsikiotis, "Fast power charging strategy for EV/PHEV in parking campus with deployment of renewable energy," in IEEE International Workshop on Computer Aided Modeling and Design of Communication Links and Networks (CAMAD), Dec. 2014, pp. 370-374.

[13] M. Wang, H. Liang, R. Zhang, R. Deng, and X. Shen, "Mobility-aware coordinated charging for electric vehicles in VANET-enhanced smart grid," IEEE Journal on Selected Areas in Communications, vol. 32, no. 7, pp. 1344-1360, Jul. 2014.

[14] S. Mehar, S. Zeadally, G. Remy, and S. Senouci, "Sustainable transportation management system for a fleet of electric vehicles," IEEE Transactions on Intelligent Transportation Systems, vol. 16, no. 3, pp. 1401-1414, Jun. 2015.

[15] E. de Souza e Silva, D. R. Figueiredo, and R. M. M. Leão, "The TANGRAM II integrated modeling environment for computer systems and networks," ACM SIGMETRICS Performance Evaluation Review, vol. 36, no. 4, pp. 64-69, 2009.

[16] Am-smart-erdam city, "The municipality of Amsterdam is expanding the charging network for electric vehicles with six fast chargers," Oct. 2015. [Online]. Available: http://amsterdamsmartcity.com/news/detail/ lang/en/id/717/slug

[17] P. Haugnel and H. H. Kvisle, "Norwegian electric car user experiences," in EVS27 International Battery, Hybrid and Fuel Cell Electric Vehicle Symposium, Sep. 2013, pp. 1-11.

\section{ACKNOWLEDGMENT}

The authors would like to thank CAPES, CNPq, and FAPERJ Brazilian agencies for partially funding this research. 


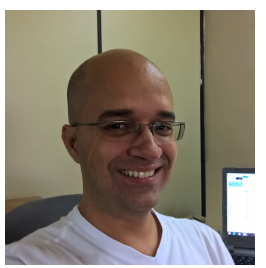

Fabio Antonio V. Pinto received his electronics engineer and M.Sc. degrees in electrical engineering from Universidade Federal do Rio de Janeiro (UFRJ), Brazil, in 1997 and 2001, respectively. He is currently D.Sc student of Electric Engineering Dept. of COPPE. Since February 2014 he has been an assistant professor with Universidade Federal do Estado do Rio de Janeiro. His major research interests are in the areas of electric vehicles and vehicular networks. Fabio has been a student member of IEEE since 2014.

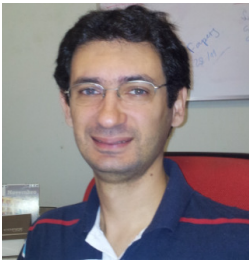

Luís Henrique M. K. Costa received his electronics engineer and M.Sc. degrees in electrical engineering from Universidade Federal do Rio de Janeiro (UFRJ), Brazil, in 1997 and 1998, respectively, and the Dr. degree from Université Pierre et Marie Curie (Paris 6), Paris, France, in 2001. Since August 2004 he has been an associate professor with COPPE/UFRJ. His major research interests are in the areas of routing, wireless networks, vehicular networks, and future Internet. Luís has been a member of IEEE COMSOC and ACM since 2001.

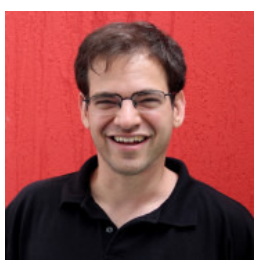

Daniel S. Menasché received his Ph.D. in computer science from the University of Massachusetts at Amherst in 2011. Currently, he is an Assistant Professor in the Computer Science Department at the Federal University of Rio de Janeiro, Brazil. His interests are in modelling, analysis and performance evaluation of computer systems. He co-authored papers that were awarded best paper at Globecom'07, CoNEXT'09, INFOCOM'13 and ICGSE'15.

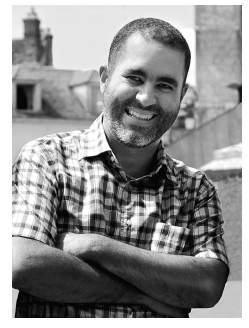

Marcelo Dias de Amorim is a research director at the National Center for Scientific Research (CNRS) and member of the computer science laboratory (LIP6) of UPMC Sorbonne Universités, France. His research interests focus on the design and evaluation of mobile systems. For more information, visit http://www-npa.lip6.fr/ amorim. 